\title{
Avulsion Fractures around the Knee and their Importance
}

\author{
Sravan Kumar Marupaka ${ }^{1}$, Shweta Kataria ${ }^{2}$, Ravikanth Jakkani ${ }^{3}$, Venkateshwarlu Jampala ${ }^{4}$, Shamsunder \\ Chavva $^{5}$ \\ ${ }^{1}$ Associate Professor, Department of Radiology, Princess Esra Hospital, DCMS, Hyderabad, 2Junior Resident, Department \\ of Radiology, Yashoda Hospitals, Malakpet, Hyderabad, ${ }^{3} \mathrm{HOD}$, Department of Radiology, Yashoda Hospitals, Malakpet, \\ Hyderabad, ${ }^{4}$ Professor and HOD, Department of Radiology, Princess Esra Hospital, DCMS, Hyderabad, ${ }^{5}$ Professor and HOD, \\ Princess Esra Hospital, DCMS, Hyderabad, India
}

Corresponding author: Sravan Kumar Marupaka, Plot NO 364, 3- 7- 62, South End Park, Mansoorabad, L B Nagar, Hydearbad -500068 , India

DOI: http://dx.doi.org/10.21276/ijcmsr.2019.4.3.54

How to cite this article: Sravan Kumar Marupaka, Shweta Kataria, Ravikanth Jakkani, Venkateshwarlu Jampala, Shamsunder Chavva. Avulsion fractures around the knee and their importance. International Journal of Contemporary Medicine Surgery and Radiology. 2019;4(3):C241-C247.

\section{A B S T R A C T}

Introduction: Avulsion fracture involves pulling off of a bone fragment from the attachment site of a ligament, tendon or joint capsule. Our study evaluated MRI findings associated with avulsion fractures around the knee.

Material and methods: This is a retrospective analytical study of avulsion fractures around the knee. This study has clearance from institutional review board. Informed consent was not needed. There are $62 \mathrm{MRI}$ studies including 51 male and 11 female patients between 14 to 73 years of age. MRI studies of patients with avulsion fractures around the knee were analyzed for associated bone and soft tissue injuries.

Results: PCL avulsions with 30 cases was the commonest avulsion around the knee in our study. ACL avulsion noted in 18 cases. Two patients had both cruciates avulsed. There was no statistically significant difference in the medial meniscal injuries between $A C L$ and $P C L$ avulsions with a $P$ value of $0.49(P<0.05$ is significant). Lateral meniscal injuries occurred in significantly larger proportion of $A C L$ avulsions compared to $P C L$ avulsions with a $P$ value of $0.007(P<0.05$ is significant).

Conclusion: Significantly larger number of cases of lateral meniscal injuries was seen in ACL avulsions compared to that of PCL. Avulsion fractures are radiographically subtle but are associated with significant soft tissue and bone abnormalities.

Keywords: Arcuate; O’ Donoghue; Popliteofibular ligament; Segond; Terrible triad.

\section{INTRODUCTION}

Avulsion fracture is so called when a bone fragment is pulled away from the attachment site of a ligament, tendon or joint capsule. Radiographs are the first imaging modality used to assess the trauma patients and the radiographic findings in avulsion fractures are very subtle like small bony fragment adjacent to the site of normal attachment of ligaments, tendons etc., in spite of other associated soft tissue and bony injuries of variable proportion of severity. ${ }^{1}$ Other imaging modalities such as CT or preferably MRI are necessary to evaluate the complete extent of injury and radiographic evidence of avulsion fractures serves as an indication for the same. ${ }^{2,3}$ They can be acute due to violent trauma or eccentric muscular contraction or chronic due to repetitive minor trauma or chronic overuse. ${ }^{4} \mathrm{We}$ are dealing only with acute avulsion fractures in this article. It is our endeavor to collect various avulsion fractures around the knee to evaluate for the associated injuries and their statistical significance and to correlate with the incidence described in the literature. Some review of literature and historical perspective of Segond fracture and O'Donoghue triad are also described in this article.

\section{MATERIAL AND METHODS}

We collected MRI studies of 62 patients with avulsion fractures around the knee joint retrospectively from our archives. It includes $51 \mathrm{male}$ patients and $11 \mathrm{female}$ patients ranging from 14 to $73 y r s$ of age.

All the MRI evaluations in this study were done in a tertiary care hospital on a 8 channel 1.5 Tesla superconducting magnet (Essenza, Siemens, Germany). PDFS FSE (TR 2800 , TE 27, Slice thickness $3.5 \mathrm{~mm}$ with a gap of $1.3 \mathrm{~mm}$ ) and T1 FSF $(827 / 13 / 3.5 \mathrm{~mm} / 1.3 \mathrm{~mm})$ sagittal, STIR coronal $(2990 / 38 / 4 \mathrm{~mm} / 1 \mathrm{~mm})$ and PDFS FSE axial $(4280 / 42 / 4.5 \mathrm{~mm} / 2.0 \mathrm{~mm})$ sequences were performed in all cases in addition to other sequences. 8 channel knee coil was used with a field of view (FOV) of $18-20 \mathrm{cms}$. No specific preparation or IV contrast was necessary in all the cases. Knee was examined in an extended relaxed position with an external rotation of about $5^{\circ}$.

Anterior (ACL) and posterior cruciate ligament (PCL) avulsions are assessed for associated meniscal and collateral ligament tears. Meniscal tears are evaluated with Chi square test for statistical significance

Only definite meniscal tears are taken into consideration. 
This means morphological abnormality in the meniscus or abnormal signal crossing the articular surface in more than one section, either in a single plane or in one section each on sagittal and coronal planes (Two slice touch rule).

We had cases of ACL, PCL, MCL (medial collateral ligament) avulsions, Segond and arcuate fractures in addition to a patellar sleeve avulsion and popliteus tendon avulsion. Representative radiographs and MR images are shown here. All these cases are evaluated for the incidence of associated meniscal, cruciate and collateral ligament injuries and other fractures and correlated with that in the literature.

\section{RESULTS}

Out of 62cases, PCL avulsion was the commonest occurring in 30 cases. ACL avulsion occurred in 18 cases. Two cases had both ACL and PCL involvement.

Among the associated injuries, meniscal involvement was the commonest followed by collateral ligament injuries which are significant only in few cases.

In 18cases with ACL avulsion, definite medial meniscal tears are seen in 6cases i.e., $1 / 3 \mathrm{rd}$ and lateral meniscal definite tears in $2 / 3$ rds i.e., 12 cases. Both lateral and medial menisci had definite tears in 3 cases of ACL avulsions and 4 cases of PCL avulsions.

PCL avulsions had medial meniscal tears in 13 out of 30 cases i.e., $43 \%$ and lateral meniscal injuries in $27 \%$ i.e., 8 cases

Chi square test demonstrates no significant association ( $p$ value of 0.493 ) between the proportion of medial meniscal injuries in ACL and PCL avulsions. However, lateral meniscal injury occurred in significantly larger proportion in case of ACL avulsions compared to PCL avulsion injuries with a $p$ value of 0.007 (less than 0.05 is significant).

It was always the posterior horn which was torn in cases of medial meniscal injuries in both ACL and PCL avulsions. Though posterior horn involvement is seen in higher number of cases, anterior horn is injured in more than $50 \%$ of cases of lateral meniscal injuries in ACL avulsions. Anterior horn is involved in larger proportion than posterior horn of lateral

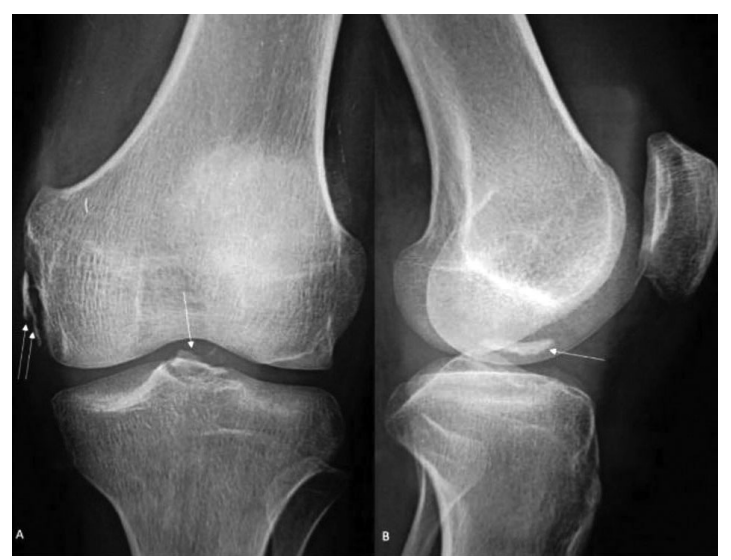

Figure-1: Combined avulsion of medial collateral ligament (MCL) and ACL. Radiograph of knee AP (A) and lateral (B) reveal bone fragment at the site of attachment of deep component of MCL (double arrow in A). Bone fragment can also be seen at the attachment site of ACL to the tibial spine (single arrow in both $\mathrm{A}$ and $\mathrm{B}$ ). This combination along with meniscal tears forms the terrible triad. meniscus in PCL avulsions.

No statistically significant association was noted between cruciate avulsions and collateral ligament injuries.

Majority of collateral ligament injuries are grade I changes with only one complete tear in the form of avulsion of MCL

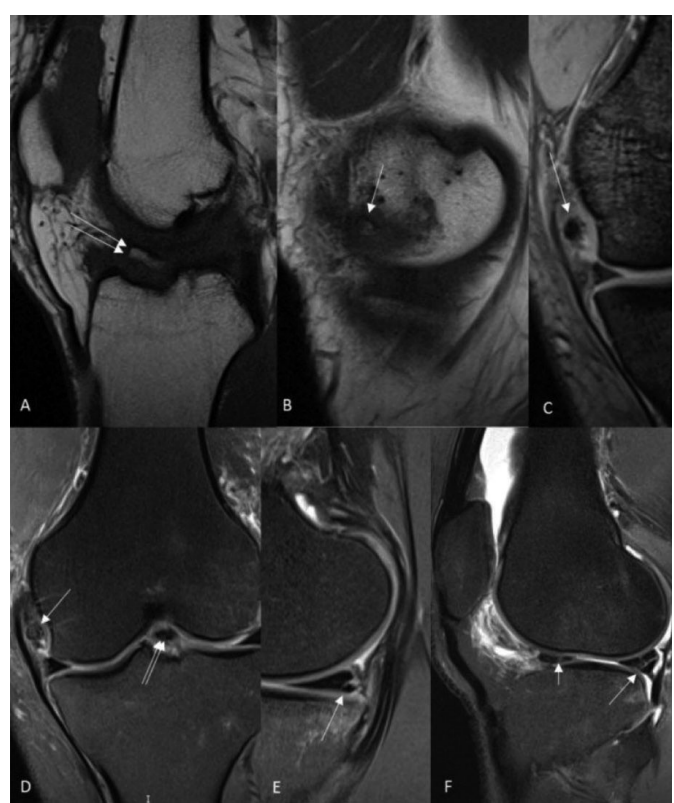

Figure-2:Unhappy and terrible Quartet.(A)T1 FSE sagittal image reveals grade III avulsion of ACL with separation of the bone fragment (double arrow) from the site of attachment. (B) T1 FSE sagittal, (C) T2 Medic coronal and (D) PD FS FSE coronal reveal avulsed bone Fragment (single arrow) from the site of attachment of meniscofemoral component of deep segment of medial collateral ligament. Double arrow in (D) denotes avulsed ACL. (E) PD FS FSE sagittal image reveals tear of posterior horn of medial meniscus (arrow). (F) PD FS FSE sagittal image reveals complex tear of posterior horn of lateral meniscus (long arrow) and vertical tear of anterior horn (short arrow). Laxity of patellar tendon can also be seen.

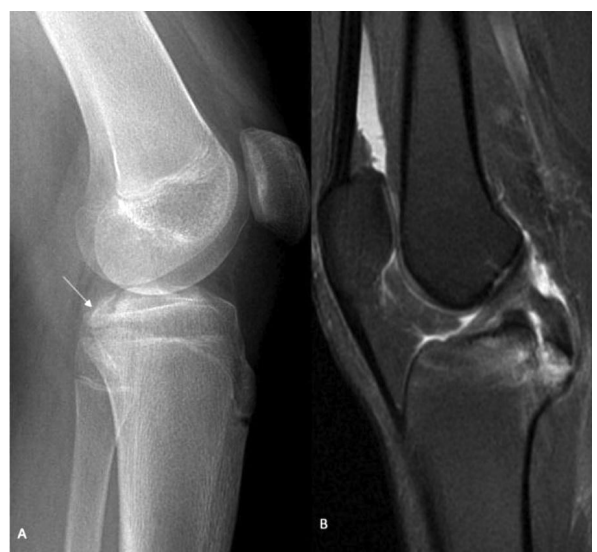

Figure-3: Posterior cruciate ligament avulsion (PCL). (A) Radiograph of knee lateral view reveals bone fragment at the site of attachment of PCL to the posterior rim of tibia in the midline. (B) PD FS FSE sagittal image reveals pulling off of bone fragment from the attachment of PCL with marrow edema in the separated fragment and adjacent tibia. Thickening of PCL. 
in case of ACL avulsion injury. One case of partial MCL tear each was noted in ACL and PCL avulsions and there were 2 cases of partial lateral collateral ligament tears in 30 patients with PCL avulsion.

Apart from 2 cases of combined ACL and PCL avulsions, there was no evidence of PCL tear in cases with ACL avulsion.

Out of 30cases for PCL avulsions, significant ACL injury

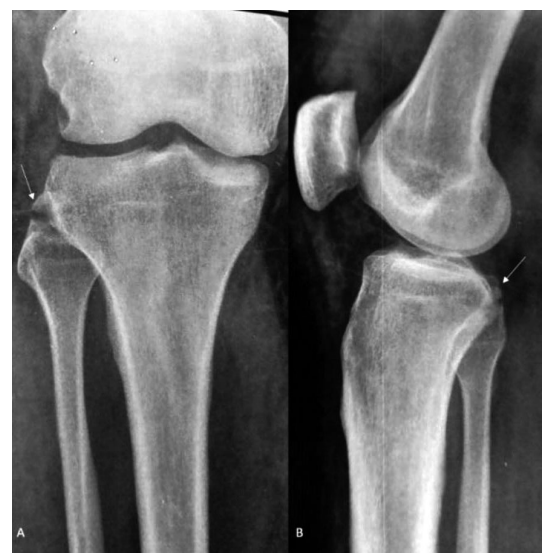

Figure-4: Arcuate fracture. Radiograph of the knee AP (A) and lateral (B) reveal avulsion of the tip of the fibula with horizontal orientation of the fragment. Indicates posterolateral corner injury.

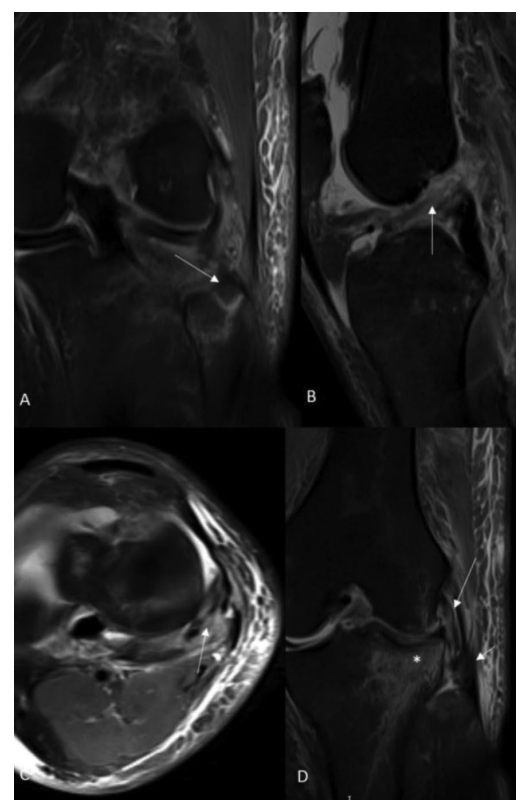

Figure-5: Arcuate fracture with severe posterolateral corner injury. (A) PD FS FSE coronal image shows fracture of the fibular styloid process (arrow). (B) PD FS FSE sagittal image reveals complete rupture of ACL (arrow). (C) PD FS FSE axial image reveals thickening and increased signal of popliteus tendon suggesting partial tear (arrow). (D) PD FS FSE coronal image reveals contusion of the lateral tibial condyle (star). Fracture lines seen on T1WI (not shown here). Thickening and increased signal of popliteus tendon (long arrow) and lateral collateral ligament (LCL - short arrow) suggesting partial tears. Arcuate fracture with partial tears of popliteus tendon and LCL along with ACL injury makes it severe posterolateral corner injury. was noted in 5 cases i.e., $16 \%$ including 2 avulsions as noted previously and one complete tear and 2 high grade partial tears. Low grade partial tears were noted in 7 cases.

Four cases of MCL avulsion, 4 cases of Segond fracture and 5 cases of arcuate fracture were noted. One case had inferior patellar avulsion and one had popliteus tendon avulsion.

Two out of four MCL avuslions had only the deep portion avulsed. One of them had associated arcuate fracture. Three MCL avulsion cases were associated with high grade or more severe tear of ACL. Remaining one was an isolated case of MCL avulsion.

One of them with deep meniscofemoral avulsion of MCL was associated with ACL avulsion and medial meniscal tear

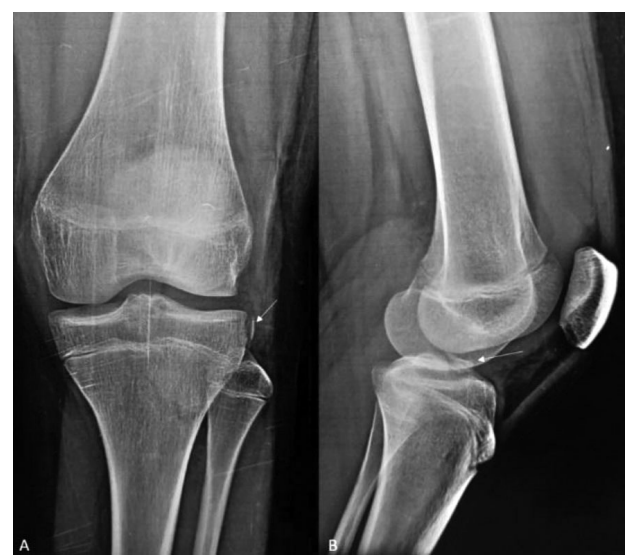

Figure-6: Segond fracture with ACL avulsion. (A) AP radiograph of knee joint reveals bone fragment from the lateral aspect of tibial Plateau just below the level of articular surface. (B) Lateral view of knee joint of the same patient reveals avulsed bone fragment from the anterior tibial spine at the site of attachment of anterior cruciate ligament. Case illustrates the strong association of Segond fracture with ACL avulsions. However contrary to the traditional view, Segond fracture is no longer considered pathognomonic for ACL avulsion.

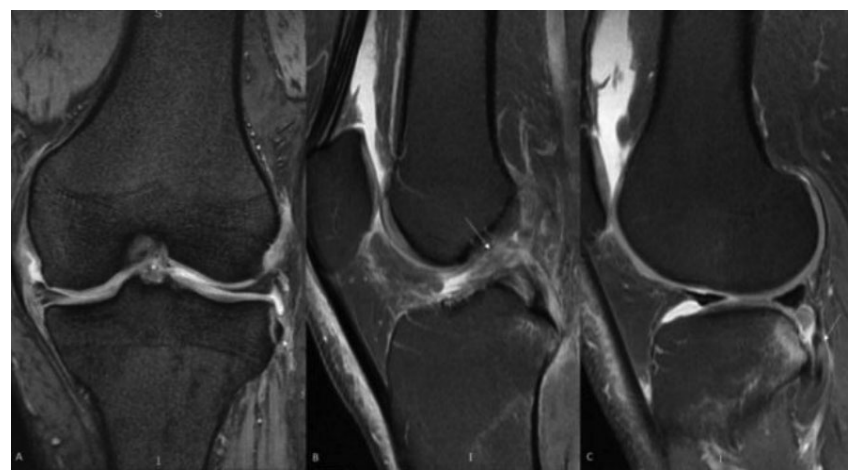

Figure-7: Segond fracture with ACL rupture and posterolateral corner injury. (A) T2 GRE coronal reveals avulsion of the lateral rim of tibial plateau -Segond fracture (arrow). (*) represents torn ACL. (B) PD FS FSE sagittal image reveals complete discontinuity of ACL. (C) PD FS FSE sagittal image reveals contusion of posterolateral portion of tibia. Thickening and increased signal intensity of popliteus tendon (arrow) - partial tear. Segond's fracture is significantly associated with ACL and posterolateral corner injuries which should always be evaluated for with MRI in any Segond fracture 
in the posterior horn. Both anterior and posterior horns of lateral meniscus also are torn - the unhappy and deadly quartet. The other two cases of combined ACL and MCL injuries don't have associated meniscal injuries.

There were four cases of Segond fracture. One of them was an isolated Segond in the sense that it was not associated with any ligamentous or meniscal injuries, though there were associated fractures of fibular head and medial femoral condyle leading to posterolateral instability.

Other three had complete tears of ACL. One of them had complete MCL tear and only one case of Segond fracture had meniscal injuries in the form of definite tear of the posterior horn of both medial and lateral meniscus. Posterolateral capsular avulsion of tibial plateau was seen in one case.

We had five cases of arcuate fractures with posterolateral corner injury of variable severity. There was associated complete ACL injury in three cases, one with avulsion. There was one case of complete PCL and two cases of MCL tear. Meniscus was involved in only two cases and it is the posterior horn of lateral meniscus in both cases.

There was a case of severe PLC injury in our series associated with avulsion of popliteus tendon from its femoral attachment. It had complete tears of both cruciate ligaments and that of lateral collateral ligament in addition to contusion of biceps femoris. It has bone contusion of anteromedial tibia corresponding to the commonest mechanism in PLC injuries.

There was no associated injury to the cruciate and collateral ligaments in the only case of patellar sleeve avulsion in our series. Posterior horn of medial meniscus had definite tear. Two cases had avulsion of posterolateral tibial plateau. One of them had Segond fracture and the other had arcuate fracture.

\section{DISCUSSION}

ACL avulsions occur more commonly in children than in adults because the apophysis is weaker than the ligament itself. They are usually isolated injuries in children. ${ }^{2}$ Pivot shift injury which involves valgus stress and internal rotation in a flexed knee and hyperextension injury are common mechanisms for ACL tears including avulsions in adults. Unlike in children, adult ACL avulsions are usually associated with bone contusions and other soft tissue injuries. ${ }^{2,5}$ Contusions heal without any sequelae but when associated with linear hypointense fracture lines, are associated with chondral injuries on follow up in as many as $50 \%$ of cases with a potential for early degenerative arthritis. Occult fracture is also an important risk factor for less than optimal clinical outcome after ACL reconstruction surgery. ${ }^{6,7}$ Our study however does not include analysis of bone contusion patterns in avulsion fractures.

Meyers and Mc Keever classified ACL avulsions into four types. ${ }^{8,2} \mathrm{ACL}$ avulsions are more common at the tibial insertion. ${ }^{1}$

Other soft tissue injuries associated with ACL tears in pivot shift injuries are posterolateral corner injuries, medial collateral ligament tears and tears of posterior horn of medial and lateral meniscus. In hyperextension injuries, there can be PCL tears in addition to posterolateral corner injuries and meniscal tears. ${ }^{5}$
Incidence of meniscal tears in ACL deficient knees varies between different studies. It is reported to be $41-82 \%$ in acute ACL tears and 58-100\% in chronic tears according to a meta-analysis by Bellabarba et al in 1997. ${ }^{9,10}$ It is quoted to be about $16-82 \%$ in acute and upto $90 \%$ in chronicACL injuries in astudy by Al Saran et al in 2004. ${ }^{11}$ Hagino et al found this to be $72 \%$ in acute and $85 \%$ in chronic ACL tears. $^{12}$

In our study meniscal tear was found in all cases of ACL avulsion. Lateral meniscus was involved in two thirds and medial meniscus in one third. Both menisci were worn in 3 out of 18 cases ACL avulsion. In contrary to this, the incidence of medial and lateral meniscal involvement was not statistically significant in a previous study of 575 cases of meniscal tears associated with ACL deficiency by Smith and Barrett in 2001. ${ }^{13}$ Posterior horn was involved in all our cases of medial meniscal tear and in 7 out of 12 cases (56\%) of lateral meniscal tear. Smith and Barrett also showed similar results with involvement of posterior horn in $99 \%$ of medial meniscal tears and $88 \%$ of lateral meniscal tears. ${ }^{13}$

MCL avulsions and injuries are common after clip injury due to valgus stress in flexion. It is a contact injury usually occurring in American football players with contusion in the lateral femoral condyle due to direct blow. ${ }^{5}$ ACL injury should always be suspected in grade III MCL injuries with valgus laxity in extension and an MRI examination is indicated in such cases (Fig 1). ${ }^{14}$

Excessive valgus stress and external femoral rotation which occurs with flexion and internal rotation of tibia results in synergistic stress on MCL and ACL resulting in combined injury along with meniscocapsular separation or peripheral vertical tear of medial meniscus due to relative lack of mobility of the medial meniscus and its attachment to the deep MCL. ${ }^{15}$ Lateral meniscus being relatively mobile is theoretically less prone to injury than the medial meniscus. ${ }^{16}$ O'Donoghue in 1950 popularised the unhappy triad of knee injury consisting of injury of medial collateral ligament, anterior cruciate ligament and the medial meniscus, first described by Campbell in 1939. 17,18,19 Combined ACL and MCL tears, on arthrotomy syudies were associated with medial meniscal tears in 30\%, lateral meniscal tears in $20 \%$ and combined injuries in 3\% cases. ${ }^{20}$ However arthroscopic evaluation by others revealed that in combined ACL and MCL tears, a lateral meniscal tear can be seen in $80 \%$ cases whereas medial meniscal tear was found in only $25 \%$ cases that to never in isolation. Vertical tear with a horizontal component at and central to the popliteal hiatus was the commonest pattern of lateral meniscal injury in this triad occurring in about $50 \%$ cases. Such a meniscal tear should therefore alert the physician to the possibility of terrible triad even in cases of remote injuries. ${ }^{21,22}$ Present study includes one such a case of unhappy or deadly quartet (Fig 2) with avulsion of ACL and deep meniscofemoral component of MCL and both medial and lateral meniscal tears.

PCL avulsions are commonly seen after motor cycle accidents or dashboard injuries and hyperextension injuries. Tibial avulsions of PCL are due to motor vehicle accidents in $68.4 \%$ of cases and out of these $59 \%$ are due to motor cycle accidents. ${ }^{23}$ They are more common in countries like India 
and China than in the western world because use of motor cycles is more in these countries. Like ACL avulsions, PCL avulsions are also more common in children than in adults. However femoral side avulsions of PCL are more common than tibial avulsions. ${ }^{4,24}$

PCL arises from the lateral aspect of medial femoral condyle and inserts 1.0 to $1.5 \mathrm{cms}$ below the posterior rim of tibial plateau. ${ }^{4}$ That is why PCL avulsions are not visualized on lateral radiographs unless the displacement is more than $1.0 \mathrm{~cm}$ (Fig 3). ${ }^{25}$

Isolated PCL injuries are rare. Multiligamentous injuries involving ACL, posterolateral and posteromedial corners are more common. ${ }^{26,27}$

Exact incidence of meniscal injuries is not known in PCL avulsions but are seen in about $30 \%$ cases of isolated PCL tears. Anterior horn of lateral meniscus is involved more often due to common mechanism of injury. In our study also, the anterior horn of lateral meniscus was injured in significantly more number of cases than the posterior horn. However there was no significant difference between medial and lateral meniscal involvement. . $^{27,24}$

Associated ACL injuries are seen in $47 \%$ cases of PCL injuries. In our study it is $16 \%$. MCL injuries occurred in $30 \%$ and posterolateral corner injuries in $26 \%$. Combined injuries are more common with intrasubstance PCL injuries than with PCL avulsions. Isolated PCL avulsions are more common than isolated intrasubstance injuries. ${ }^{24}$

Arcuate fractures are horizontally oriented avulsions of fibular styloid process due to injury to the arcuate complex which includes popliteofibular, fabellofibular and arcuate ligaments (Fig 4). ${ }^{28,29}$ Arcuate complex along with lateral collateral ligament, popliteus tendon and muscle and biceps femoris tendon are the stabilisers of posteroilateral corner which prevent posterior translation, varus angulation and external rotation of tibial plateau relative to femur. Popliteofibular ligament or fibular head of popliteus muscle is considered the most important static stabilizer of the posterolateral corner according to a biomechanical study. ${ }^{30}$

Most common mechanism of injury is anteromedially directed blow to the externally rotated tibia with knee in extension. $^{31,29}$

Arcuate sign indicates posterolateral corner injury (Fig 5) and is strongly associated with PCL injury. Strong association is due to the common mechanism of injury involving fibular head fracture and PCL tear. ${ }^{28,29,31}$

Juhng et al. reported 19 knees presenting with an arcuate sign on radiographs. Cruciate ligament injuries were seen in 16 cases (89\%) out of which, 9 patients had combined ACL and PCL injury. Isolated ACL injury was noted in 4 cases and three had isolated PCL injury. Bone contusion or fracture was noted in the anteromedial femoral condyle or at the anteromedial tibia in all the cases. Medial meniscus was torn in $28 \%$ and $22 \%$ had lateral meniscus tear. Tear of the posterolateral capsule was reported in $67 \%$ of the cases. All patients had a joint effusion. ${ }^{32}$

If unrecognized, arcuate farcture may lead to chronic instability, accelerated osteoarthritis and failure of cruciate ligament reconstruction surgeries. ${ }^{33}$

Significant posterolateral corner instability should be suspected when complete tear of any two of the three static posterolateral stabilizers or any one associated with cruciate ligament injuries. ${ }^{33}$

Common peroneal nerve is susceptible to injury in arcuate fractures. $^{34}$

In Segond fracture, a vertically oriented small bone fragment is seen adjacent to the lateral tibial condyle just below the level of articular margin. ${ }^{35}$ Traditionally it is said to be due to avulsion of lateral capsular ligament due to excessive internal rotation and varus stress and was thought to be pathognomonic for ACL tear (Fig 6). It is an extensively studied avulsion fracture, described first by Paul Segond in $1879 .{ }^{36}$ There were various descriptions of components of this lateral capsular ligament over the years. ${ }^{37,38,39}$ It is now thought to be due to avulsion of anterolateral ligament (ALL) which is found to be a distinct structure due to thickening of anterolateral capsule behind the Gerdy tubercle similar to glenohumeral ligaments of shoulder joint. ${ }^{40,41,42}$ ALL was clearly demonstrated on 3.0T MRI and through step by step dissection method. ${ }^{43,44}$ According to Claes et al. origin of ALL is from the lateral femoral epicondyle, just anterior to the origin of lateral collateral ligament and passing caudally in close association with the body of the lateral meniscus to insert onto the proximal tibia between Gerdy tubercle and fibular head. ${ }^{45}$ It is an anterolateral stabilizer of the knee.

Clinically, patients present with pain at the lateral joint line and with anterolateral rotational instability. ${ }^{46,47}$ Identification of these fractures is crucial because of high incidence of associated anterior cruciate ligament tears (75\%-100\%), meniscus tears (66\%-75\%) and posterolateral corner injuries. It is no longer considered pathognomonic for ACL tear. $^{48,35,49,38}$ However thorough clinical and radiological evaluation for ACL and posterolateral corner injuries is required in all cases of Segond fracture (Fig 7). ${ }^{50}$

In a study done by Campos et al of 17 cases of Segond fracture, ACL tears and knee joint effusions were present in 94\% of the patients, $30 \%$ had medial meniscal tears and $23 \%$ had lateral meniscus tears. Bone contusions were present in $82 \%$ of the cases, mostly in lateral tibial plateau and femoral condyle. Injuries to the PLC structures were present in 35\% cases and tears of medial collateral ligament in $35 \% .{ }^{38}$ In our study there is an example of Segond fracture without ACL tear. Same case has fracture of fibular head suggesting associated posterolateral corner injury.

Patellar sleeve avulsion fracture is an acute avulsion of cartilage along with bone fragment at the inferior pole of patella.

\section{CONCLUSION}

Avulsion fractures are radiographically subtle but are associated with significant soft tissue and bone abnormalities. Significantly larger number of cases of lateral meniscal injuries was seen in ACL avulsions compared to that of PCL. There was no statistically significant difference in the medial meniscal injuries between ACL and PCL avulsions

\section{REFERENCES}

1. Delzell PB, Schils JP, Recht MP. Subtle fractures about the knee: innocuous-appearing yet indicative of 
significant internal derangement. AJR Am J Roentgenol 1996;167(1):699-703

2. Gottsegen CJ, Eyer BA, White EA, Learch TJ, Forrester D. Avulsion fractures of the knee: imaging findings and clinical significance. Radiographics. 2008; 28(6): 1755 70

3. Venkatswamy A, Ehlingerb M, Bierrya G. Acute traumatic knee radiographs: Beware of lesions of little expression but of great significance. Diagnostic and Interventional Imaging 2014;95(6):551-60

4. J.M.Mellado A, Ramos E, Salvadó A, Camins J, Calmet A, Saurí. Avulsion fractures and chronic avulsion injuries of the knee: role of MR imaging. EurRadiol 2002;12 (3):2463-2473.

5. Timothy G, Sanders, Monica A, Medynski, John F. Feller, Keith W. Lawhorn. Bone Contusion Patterns of the Knee at MR Imaging: Footprint of the Mechanism of Injury. RadioGraphics 2000; 20 (3):S135-S151.

6. Richard Kijowski, Mamadou L. Sanogo, Kenneth S. Lee, Alejandro Muñoz del Río, Tim A. McGuine, Geoffrey S. Baer, et al. Short-term clinical importance of osseous injuries diagnosed at MR imaging in patients with anterior cruciate ligament tear. Radiology 2012; 264 (5):531-54.

7. Kaplan PA, Walker CW, Kilcoyne RF, Brown DE, Tusek D, Dussault RG. Occult fracture patterns of the knee associated with anterior cruciate ligament tears: assessment with MR imaging. Radiology 1992;183(3):835-838

8. Meyers MH, McKeever FM. Fractures of the intercondylar eminence of the tibia. J Bone Joint Surg Am 1970;52 (2):1677-1684

9. Bellabarba C, Bush-Joseph CA, Bach BR Jr. Patterns of meniscal injury in the anterior cruciate-deficient knee: a review of the literature. Am J Orthop 1997; 26 (6): 18-23.

10. Vinson EN, Gage JA, Lacy JN. Association of peripheral vertical meniscal tears with anterior cruciate ligament tears. Skeletal Radiol 2008; 37 (5): 645-651

11. Al Saran Y, Al Lhaidan A, Al Garni N, Al Aqeel M, Alomar A, Bin Nasser A, et al. Patterns of Meniscal Damage Associated with Acute ACL Rupture. J Orthopedics Rheumatol. 2014;2(1): 4.

12. Hagino T, Ochiai S, Senga S, Yamashita T, Wako M, Ando T, et al. Meniscal tears associated with anterior cruciate ligament injury. Arch Orthop Trauma Surg 2015;135(12):1701-1706.

13. Smith JP, Barrett GR. Medial and lateral meniscal tear patterns in anterior cruciate ligament-deficient knees. Am J Sports Med 2001;29 (1):415-419.

14. Phinit Phisitkul, Stan L James, Brian R Wolf, AnnunziatoAmendola. MCL Injuries of the Knee: Current Concepts Review. Iowa Orthop J. 2006;26 (5):77-90

15. Peter J. MacMahon, William E. Palmer. A Biomechanical Approach to MRI of Acute Knee Injuries. AJR 2011;197 (1):568-577.

16. Peter Jonathan Dacombe. Shelbourne's update of the O'Donoghue knee triad in a 17-year-old male Rugby player. BMJ Case Rep. 2013;2013: bcr0120125593.

17. O'Donoghue DH. Surgical treatment of fresh injuries to the major ligaments of the knee. J Bone Joint Surg 1950;32A (3):721-38

18. O'Donoghue DH. An analysis of end results of surgical treatment of major injuries to the ligaments of the knee. J Bone Joint Surg1955;37A (1):1-13.

19. Campbell WC. Reconstruction of the ligaments of the knee. Am J Surg 1939;43 (6):473-80.

20. Cerabona F, Sherman MF, Bonamo JR, Sklar J. Patterns ofmeniscal injury with acute anterior cruciate ligament tears.Am .I Sports Med 1988;16 (5):603-9.

21. Shelboume KD, Nitz PA. Knee injury triad: anterior cruciate ligament, medial collateral ligament, and lateral meniscus. Am J Sports Med 1991;19 (2):47\&7.

22. F. Alan Barber, What Is the Terrible Triad? Arthroscopy: The Journal of Arthroscopic and Related Surgery 1992;8(1):19-22.

23. Hooper PO.Management of posterior cruciate ligament tibial avulsion injuries: a systematic review. Am J Sports Med 2018;46(3):734-742.

24. Chen G, Fu WL, Tang X, Li Q, Li J. Clinical epidemiological analysis on posterior cruciate ligament injuries. ZhongguoGu Shang. 2015;28 (5):638-642.

25. Zhao J. Arthroscopic treatment of acute tibial avulsion fracture of the posterior cruciate ligament with suture fixation technique through Y-shaped bone tunnels. Arthroscopy 2006;22(2):172-81.

26. Ali M. Naraghi, Lawrence M. White. Imaging of Athletic Injuries of Knee Ligaments and Menisci: Sports Imaging Series. Radiology 2016;281:23-40.

27. Hamada M, Shino K, Mitsuoka T, Toritsuka Y, NatsuUme T, Horibe S. Chondral injury associated with acute isolated posterior cruciate ligament injury. Arthroscopy 2000;16(1):59-63.

28. Shindell R, Walsh WM, Connolly JF. Avulsion fracture of the fibula: the "arcuate sign" of posterolateral knee instability. Nebra Med J 1984;69 (3):369-371.

29. Huang GS, Yu JS, Munshi M, Chan WP, Lee CH, Chen CY, et al. Avulsion fracture of the head of the fibula (the "arcuate" sign): MR imaging findings predictive of injuries to the postero-lateral ligaments and posterior cruciate ligament. AJR Am J Roentgenol 2003;180 (3):381-7.

30. Veltri DM, Deng X-H, Torzilli PA, Maynard MJ, Warren RF. The role of the popliteaofibular ligament in stability of the human knee: a biomechanical study. Am J Sports Med 1996;24 (5):19-27.

31. Baker CL, Norwood LA,Hughston JC. Acute combined posterior cruciate and posterolateral instability of the knee. Am J Sports Med 1984;12 (5):204-208.

32. Juhng SK, Lee JK, Choi SS, Yoon KH, Roh BS, Won JJ. MR evaluation of the "arcuate" sign of posterolateral knee instability. AJR Am J Roentgenol 2002;178(3):583-8

33. Humberto G. Rosas. Unraveling the Posterolateral Corner of the Knee. RadioGraphics 2016; 36 (1):17761791.

34. Demuynck M, Zuker RM. The peroneal nerve: Is repair worthwhile? J Reconstr Microsurg 1987; 3(3):193197,199.

35. Goldman AB, Pavlov H, Rubenstein D. The Segond fracture of the proximal tibia: a small avulsion that reflects major ligamentous damage. AJR Am J 
Roentgenol 1988;151(6):1163-7

36. Segond P. Recherchescliniquesetexpérimentales sur les épanchementssanguins du genou par entorse. ProgrèsMédical 1879; 16: 297-299, 319-321, 340-341

37. Claes S, Luyckx T, Vereecke E, Bellemans J. The Segond fracture: a bony injury of the anterolateral ligament of the knee. Arthroscopy: The Journal of Arthroscopic \& Related Surgery 2014;30(11):1475-1482.

38. Campos JC, Chung CB, Lektrakul N, Pedowitz R, Trudell D, Yu J, et al. Pathogenesis of the Segond fracture: anatomic and MR imaging evidence of an iliotibial tract or anterior oblique band avulsion. Radiology 2001;219(2):381-386.

39. Humza Shaikh, ElmarHerbst, Ata Amir RahnemaiAzar, MarcioBottene Villa Albers, Jan-Hendrik Naendrup, Volker Musahl, et al. The Segond Fracture Is an Avulsion of the Anterolateral Complex. Am J Sports Medicine 2017;45(10): 2247-2252.

40. Caterine S, Litchfield R, Johnson M, Chronik B, Getgood A. A cadaveric study of the anterolateral ligament: reintroducing the lateral capsular ligament. Knee Surg Sports TraumatolArthrosc 2015;23 (1):3186-3195.

41. Jonathan D.Kosy,Ashish Soni,RamakrishnanVenkatesh, Vipul I. Mandalia. The anterolateral ligament of the knee: unwrapping the enigma. Anatomical study and comparison to previous reports. J OrthopaedTraumatol 2016;17 (6):303-308.

42. Schindler OS. Surgery for anterior cruciate ligament deficiency: a historical perspective. Knee Surg Sports TraumatolArthrosc 2012;20(1):5-47.

43. Yong Qin, Ming Li, Songcen L, Haile Pan. An anatomical and MRI assessment of the of anterolateral ligament. Biomedical Research 2018;29(9):1784-1787.

44. Diego Ariel de Lima, Camilo PartezaniHelito, Matthew Daggett, Francisco Magalhães Monteiro Neto, Lana Lacerda de Lima, José Alberto Dias Leite, et al. Anterolateral ligament of the knee: a stepby-step dissection BMC Musculoskeletal Disorders 2019;20 (6):142.

45. Claes S, Vereecke E, Maes M, Victor J, Verdonk P, Bellemans J. Anatomy of the anterolateral ligament of the knee. J Anat 2013;223 (3):321-328.

46. Stevens MA, El-Khoury GY, Kathol MH, Brandser EA, Chow S. Imaging features of avulsion injuries. Radiographics 1999;19 (1):655-672.

47. Dietz GW, Wilcox DM, Montgomery JB. Segond tibial condyle fracture: lateral capsular ligament avulsion. Radiology 1986;159 (5):467-9.

48. D. Ollat, J. P. Marchaland, L. Mathieu, O. Barbier, G. Versier. Is the Segond's fracture a reliable sign of anterior cruciate ligament (ACL) tear? A case report without associated ACL rupture. Eur J Orthop Surg Traumatol 2009;19 (4):203.

49. W N Weber, C H Neumann, J A Barakos, S A Petersen, L S Steinbach, H K Genant. Lateral tibial rim (Segond) fractures: MR imaging characteristics. Radiology 1991;180(3):731-4.

50. Cohen AP, King D, Gibbon AJ. Impingement fracture of the anterior medial tibial margin: a radiographic sign of combined posterolateral complex and posterior cruciate ligament disruption. Skeletal Radiol 2001;30 (1):114-116

\section{Source of Support: Nil; Conflict of Interest: None}

Submitted: 01-08-2019; Accepted: 24-08-2019; Published online: 21-09-2019 